\title{
Solvent Screening for Cyclopentane Purification Based on COSMO-RS
}

\author{
Lehuan $\mathrm{Wu}$, Xinyun Pu, and Yansheng Liu
}

\begin{abstract}
Separation of cyclopentane has an important significance in environmental protection and resource utilization. This work is intended to serve as a rapid way to screen mixed solvent for extractive distillation of cyclopentane using the COSMO-RS method. IDACs and VLE data of different binary systems were compared with computational results. Then the performance of mixed solvent was studied by the COSMO-RS method, including the influences of the solvent-to-feed ratio and the basic solvent and cosolvent ratio on the relative volatility. Finally, the mixed solvent was validated to be effective by the experiments.
\end{abstract}

Index Terms-COSMO-RS, cyclopentane, solvent screening, IDACs.

\section{INTRODUCTION}

As an important industrial chemical, cyclopentane is generally used as a new-type blowing agent to replace the chlorofluorocarbons (CFCs), which have serious influence on ozone layer [1], [2]. With the refrigeration industry developing rapidly, the market demand for cyclopentane is also increasing. However, the purity of cyclopentane is restricted by the separation technology. In the light hydrocarbons from gas and oil fields or cracked C5 fractions from catalytic cracking units, the content of cyclopentane is relatively high, however, neohexane (2,2-dimethyl butane) also exists, which forms azeotrope with cyclopentane. Moreover, the difference of boiling points between these two components is close to $0.5^{\circ} \mathrm{C}$. Therefore, it results in a great difficulty in cyclopentane purification.

The selection of separation technology is according to the physical or chemical difference between the two components. Due to the properties of this system, conventional distillation technology cannot be applied to achieve the goal of separation effectively. Since Hicks-Bruun et al. first noticed the mixture of cyclopentane and neohexane in petroleum products in 1939 [3], different separation methods have been used, including extraction, azeotropic distillation and extractive distillation [4, 5]. What's more, extractive distillation has superiority over extraction and azeotropic distillation in some respects, for example, the operation difficulty is lower and it has a broader range of solvent selection, so it gradually became the main method used for separation of cyclopentane and neohexane. According to the extractive distillation principle, polar solvents are more perferred. There are some solvents reported for separating this system up to now, such as

Manuscript received July 13, 2016; revised January 10, 2017.

The authors are with with the China University of Petroleum, Beijing, 102249, China (Corresponding author: Yansheng Liu; e-mail: wsuper@cup.edu.cn, zhoushan1987@hotmail.com,634660245@qq.com).
$\mathrm{N}$-( $\beta$-mercaptoehtyl)-2-pyrrolidone (NMEP), cyclohexanol (CHOL), N-methyl-pyrrolidone (NMP), or a mixture of NMEP and either CHOL or NMP, phenol and NMP+5\% water [6], [7]. Among these solvents, mixed solvent tends to be more effective than single solvent, since single solvent cannot have high selectivity and solubility simultaneously. In order to study the properties of mixed solvent, fast screening method such as COSMO-RS (conductor-like screening model for real solvents) method was applied to select the suitable solvents, due to its great predictive power and broad applicability in calculating thermodynamic properties of liquid mixtures [8], [9].

\section{INFINITE DILUTION ACTIVITY COEFFICIENTS (IDACS)}

The selectivity, i. e. the change degree of the relative volatility of the components as the solvent being added into the system, is the key factor for solvent screening. The definition of selectivity is the ratio of the relative volatilities of two key components after and before adding the solvent. So the relative volatility $\alpha_{i j}$ can be determined to be:

$$
\alpha_{i j}=\frac{y_{i} / x_{i}}{y_{j} / x_{j}}=\frac{\gamma_{i}^{\infty} P_{i}^{S}}{\gamma_{j}^{\infty} P_{j}^{S}}
$$

where $i, j$ are the light and heavy key components, respectively, $\gamma^{\infty}$ is the infinite dilution activity coefficient, and $P^{S}$ is the saturated vapor pressure of the component. From (1), it is obvious that the selectivity is related with the IDACs of two key components in the solvent. Therefore, we can obtain the selectivity of the solvents approximately as long as the IDACs of cyclopentane and neohexane in different solvents are known.

Up to now, there are many IDAC data of cycloalkanes and alkanes in different solvents reported in the reference. However, the data of cyclopentane and neohexane are deficient. As a prior prediction model, the COSMO-RS model can calculate the IDACs in a short time [10], [11], which may make up the inadequate data, though the accuracy of the model needs to be observed. Mathias found that the COSMO-RS model could capture the trend of IDACs for hydrocarbon-solvent, and studied the application of the model in the separation of $\mathrm{C} 4$ hydrocarbons [12]. According to the above study, we investigated the solvent screening for cyclopentane-neohexane extractive distillation by using the COSMO-RS model.

We calculated the IDACs of cycloalkanes and alkanes in three solvents by the COSMO-RS model, and then compared with the data in [9], as seen in Fig. 1-Fig. 3. It can be known that there exit linear relationships between the calculated 
IDAC results and the reference data of hydrocarbons in three solvents, respectively. In order to predict the unknown IDAC data, the relationships were correlated. The fitted relationships for alkane-solvent or cycloalkane-solvent IDACs in terms of the COSMO-RS predictions were shown as follows:

ACN (Acetonitrile):

$$
\begin{aligned}
& \ln \gamma_{\text {correlated }}^{\infty}(\text { alkanes })= \\
& 0.23529+0.85452 \ln \gamma^{\infty}-0.02291\left(\ln \gamma^{\infty}\right)^{2} \\
& \ln \gamma_{\text {correlated }}^{\infty}(\text { cycloalkanes })= \\
& \quad-0.53678+1.169 \ln \gamma^{\infty}-0.05122\left(\ln \gamma^{\infty}\right)^{2}
\end{aligned}
$$

DMF (N, N-dimethyl formamide):

$$
\begin{aligned}
& \ln \gamma_{\text {correlated }}^{\infty}(\text { alkanes })= \\
& 1.12054+1.44568 \ln \gamma^{\infty}-0.18313\left(\ln \gamma^{\infty}\right)^{2} \\
& \ln \gamma_{\text {correlated }}^{\infty}(\text { cycloalkanes })= \\
& -0.65862+2.63436 \ln \gamma^{\infty}-0.19138\left(\ln \gamma^{\infty}\right)^{2}
\end{aligned}
$$

NMP (N-methyl-2-pyrrolidone):

$$
\begin{aligned}
& \ln \gamma_{\text {correlated }}^{\infty}(\text { alkanes })= \\
& 0.89422+1.88665 \ln \gamma^{\infty}+0.0151\left(\ln \gamma^{\infty}\right)^{2} \\
& \ln \gamma_{\text {correlated }}^{\infty}(\text { cycloalkanes })= \\
& 1.46271+0.13743 \ln \gamma^{\infty}+0.8776\left(\ln \gamma^{\infty}\right)^{2}
\end{aligned}
$$

There are IDAC data of some hydrocarbons in ACN including cyclopentane and neohexane in [13], the COSMO-RS calculated data and the correlated data using (2) and (3) were listed in Table I, and the comparison were shown in Fig. 4. As is shown in Fig. 4, the correlated data is more accurate than the COSMO-RS calculated data, the average relative deviations are 0.24 and 21.19 , respectively.
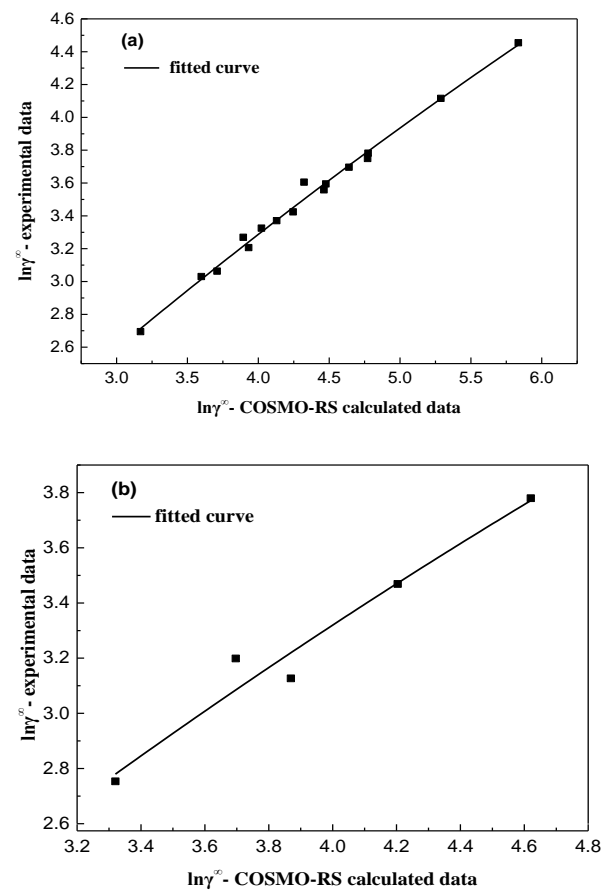

Fig. 1. Relationships of $\ln \gamma^{\circ}$ of alkanes (a) and cycloalkanes (b) in ACN between the predicted and experimental data [9] at $25^{\circ} \mathrm{C}$
Therefore, it's a convenient and accurate way to calculate IDACs of hydrocarbons in solvents by using the empirical relationships.

With the help of these empirical relationships, we can obtain much more accurate IDAC data as long as we calculate the IDACs of cyclopentane and neohexane in three solvents by using the COSMOtherm software. Therefore, the relative volatilities of cyclopentane-neohexane system in three preselected solvents were given from (1), listed in Table II. As is known from Table II, three solvents that affect the relative volatilities of cyclopentane-neohexane system are in the following order: $\mathrm{DMF}>\mathrm{NMP}>\mathrm{ACN}$.

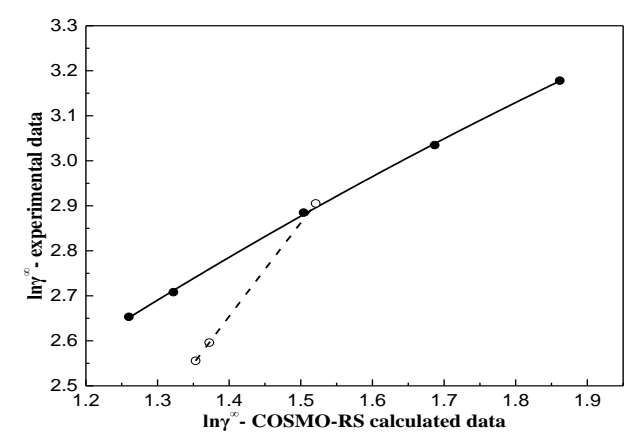

Fig. 2. Relationships of $\ln \gamma^{\circ}$ of alkanes and cycloalkanes in DMF between the predicted and experimental data [9] at $25^{\circ} \mathrm{C}$. ( $\mathrm{\circ}$ ) comparison for cycloalkane-solvent; $(\bullet)$ comparison for alkane-solvent; dashed line: fitted curve for cycloalkane-solvent; solid line: fitted curve for alkane-solvent.

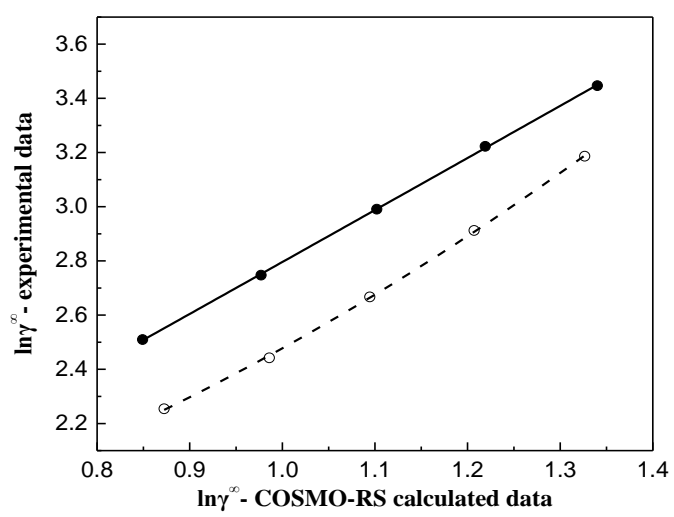

Fig. 3. Relationships of $\ln \gamma^{\circ}$ of alkanes and cycloalkanes in NMP between the predicted and experimental data at $25{ }^{\circ} \mathrm{C}$. (0) comparison for cycloalkane-solvent; $(\bullet)$ comparison for alkane-solvent; dashed line: fitted curve for cycloalkane-solvent; solid line: fitted curve for alkane-solvent.

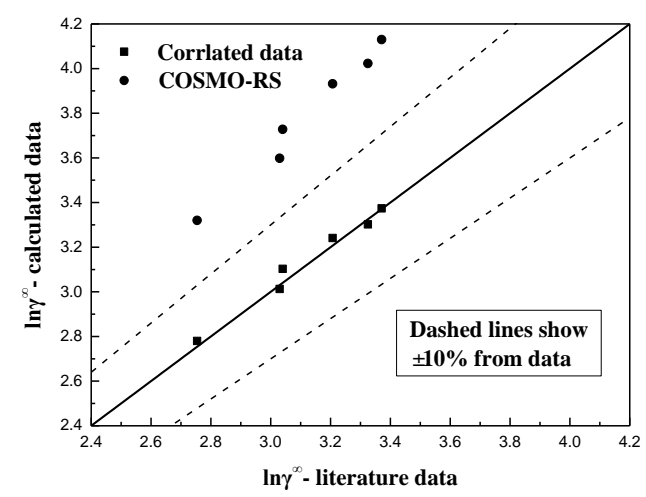

Fig. 4. Comparison of the COSMO-RS calculated data and the correlated data of $\ln \gamma \infty$ in $\mathrm{ACN}$ at $25^{\circ} \mathrm{C}$. 
TABLE I: COMPARISON OF THE COSMO-RS CALCULATED DATA AND THE Correlated Data of LN(IDAC) IN ACN AT $25{ }^{\circ} \mathrm{C}$

\begin{tabular}{|c|c|c|c|c|c|}
\hline \multirow{2}{*}{ Component } & \multicolumn{3}{|c|}{$\ln \gamma^{\infty}$ in $\mathrm{ACN}$ at $25^{\circ} \mathrm{C}$} & \multicolumn{2}{|c|}{$\begin{array}{c}\text { Relative deviation } \\
(\%)\end{array}$} \\
\hline & $\begin{array}{l}\text { Data } \\
\text { in [9] }\end{array}$ & $\begin{array}{c}\text { COSMO } \\
\text {-RS } \\
\end{array}$ & $\begin{array}{c}\text { Correlate } \\
\text { d data }\end{array}$ & $\begin{array}{c}\text { COSMO } \\
\text {-RS }\end{array}$ & $\begin{array}{c}\text { Correlate } \\
\text { d data }\end{array}$ \\
\hline isopentane & 3.03 & 3.6 & 3.01 & 18.74 & -0.66 \\
\hline n-pentane & 3.06 & 3.73 & 3.09 & 21.90 & 0.98 \\
\hline $\mathrm{CP}$ & 2.75 & 3.32 & 2.78 & 20.73 & 1.09 \\
\hline $\mathrm{NH}$ & 3.21 & 3.93 & 3.24 & 22.43 & 0.93 \\
\hline $\begin{array}{l}\text { 2-methyl } \\
\text { pentane }\end{array}$ & 3.37 & 4.13 & 3.37 & 22.55 & 0.00 \\
\hline $\begin{array}{l}\text { 2-methyl } \\
\text { pentane }\end{array}$ & 3.33 & 4.02 & 3.30 & 20.72 & -0.90 \\
\hline \multicolumn{4}{|c|}{ Average relative deviation $(\%)$} & 21.19 & 0.24 \\
\hline
\end{tabular}

TABLE II: THE RELATIVE VOLATILITY OF CYCOLPENTANE (CP) AND NEOHAXANE (NH) SYSTEM AT $25^{\circ} \mathrm{C}$

\begin{tabular}{cccccc}
\hline \hline \multirow{2}{*}{ Solvent } & \multicolumn{3}{c}{$\gamma^{\infty}$} & \multicolumn{3}{c}{ Vapor pressure (kPa) } & \multirow{2}{*}{$\alpha$} \\
\cline { 2 - 4 } & $\mathrm{CP}$ & $\mathrm{NH}$ & $\mathrm{CP}$ & $\mathrm{NH}$ & \\
\hline ACN & 16.11 & 25.57 & & & 1.60 \\
$\mathrm{DMF}$ & 8.46 & 15.38 & 42.33 & 42.74 & 1.84 \\
$\mathrm{NMP}$ & 7.69 & 12.73 & & & 1.67 \\
\hline \hline
\end{tabular}

\section{MIXED SOLVENT}

\section{A. Binary VLE Predictions}

In order to determine the accuracy of the COSMO-RS model, the experimental data of binary VLE were compared with the COSMO-RS predicted results. We did the VLE experiments for ethanol + cyclopentane and ethanol + neohexane systems by using the CP-I dual circulation vapor-liquid equilibrium still. The diagram of the apparatus is shown in Fig. 5.

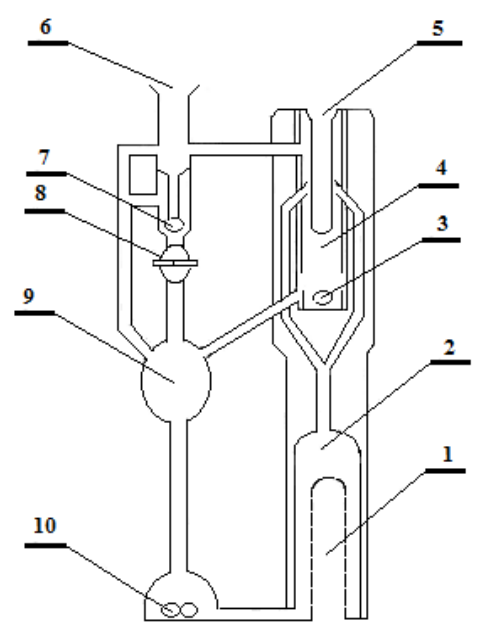

Fig. 5. Diagram of CP-I dual circulation vapor-liquid equilibrium still: 1. heating coil; 2. boiling chamber; 3. liquid-phase sampling port; 4. Equilibrium chamber; 5. thermometer well; 6 . connected to the condenser; 7. vapor-phase sampling port; 8 . valve; 9. mixing chamber; 10 . magnetic stirrer.

Fig. 6 shows that the temperature minimum azeotropic behaviors were both exiting in the experimental results and the COSMO-RS prediction for the cyclopentane and ethanol system. The predicted mole fraction of cyclopentane at the azeotropic point is 0.851 , compared with the experimental data 0.855 , the relative deviation of the COSMO-RS model is
$0.452 \%$. Besides, the difference of azeotropic temperature between the predicted and experimental data is close to $1.3 \mathrm{~K}$, with the relative deviation being $0.409 \%$. In addition, the average relative deviation of mole fraction of cyclopentane in the vapor phase is $1.77 \%$, which shows that the COSMO-RS predictions for cyclopentane-ethanol system are in good agreement with the experimental results.

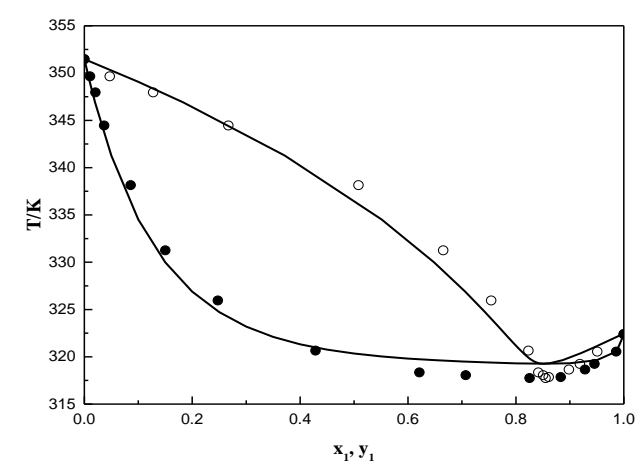

Fig. 6. VLE data of cyclopentane (1) + ethanol (2) system at $101.3 \mathrm{kPa}$. (०) T-y1 experimental data; $(\bullet)$ T-x1 experimental data; Solid lines: COSMO-RS calculations.

Fig. 7 shows that neohexane and ethanol also form a temperature minimum azeotrope. The predicted azeotropic composition of neohexane is 0.8325 (mole fraction), while the experimental value is 0.8582 , thus the relative deviation is $2.995 \%$. The difference of azeotropic temperature between the predicted and experimental data is close to $1.5 \mathrm{~K}$, with the relative deviation being $0.475 \%$. Moreover, the mean relative deviation of mole fractions of neohexane in the vapor phase between the experimental and predicted values is $5.13 \%$, so the deviation of the COSMO-RS model is also acceptable.

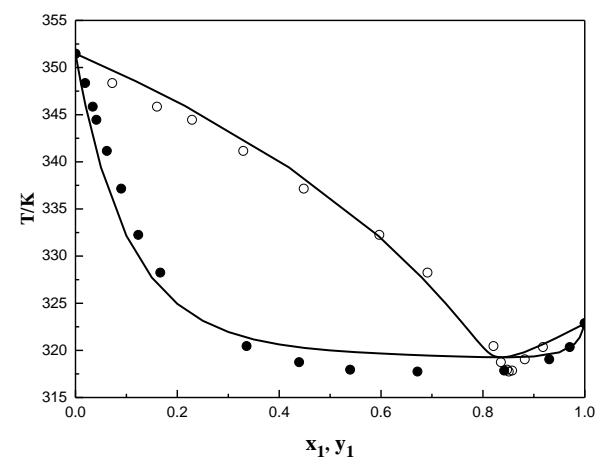

Fig. 7. VLE data of neohexane (1) + ethanol (2) system at 101.3kPa. (o) T-y1 experimental data; $(\bullet) \mathrm{T}$-x 1 experimental data; Solid lines: COSMO-RS calculations.

To study the performance of mixed solvent for separation of cyclopentane-neohexane system, the COSMO-RS model was applied to investigate the variations of relative volatility as the mixed solvent (DMF + cyclohexanol) was added.

\section{B. Quaternary VLE Predictions}

DMF was chose to be the basic solvent due to its high selectivity, and cyclohexanol was the cosolvent. In the process of COSMO-RS calculations, the cyclopentane and neohexane mass ratio was set to $4: 1$, and the mass fraction of DMF in mixed solvent changed from 0.5 to 0.9 . In addition, the solvent and feed mass ratio varied from 2:1 to 7:1. Fig. 6 
shows the influences of different factors on relative volatility of cyclopentane-neohexane system. It can be known that themixed solvent can apparently alter the relative volatility. With the solvent-to-feed ratio increasing, the relative volatility increases gradually. However, the gradient of variation of relative volatility becomes small, thus the relative volatility tends to be constant.

While mixed solvent was added to the system, the relative volatility of cyclopentane and neohexane system rises with the mass fraction of DMF in mixed solvent increasing, as shown in Fig. 8. It can be explained from the ratio of dielectric constant to molecular weight $\varepsilon / M$, as the $\varepsilon / M$ of DMF and cyclohexanol is 0.5233 and 0.1637 [14], respectively. Therefore, on the condition that the total mass of mixed solvent is constant, as the cyclohexanol molecules gradually replace the DMF molecules, the polarity of mixed solvent diminishes, which leads to the reduction of the relative volatility of cyclopentane-neohexane system.

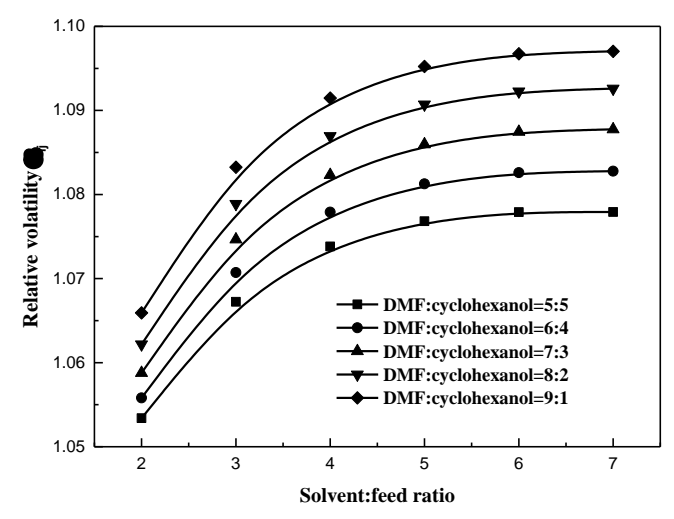

Fig. 8. The effect of the solvent-to-feed ratio and the basic solvent and cosolvent ratio on the relative volatility using DMF + cyclohexanol mixed solvent.

\section{Quaternary VLE Experimental Results}

According to the predictions of the COSMO-RS method, the suitable ratio of solvent-to-feed or basic solvent to cosolvent can be easily determined. We finally obtained the VLE data of quaternary system of cyclopentane-neohexane-DMF-cyclohexanol from the experimentation, as the solvent-to-feed ratio changed from $2: 1$ to $6: 1$, the cyclopentane and neohexane mass ratio was $4: 1$, and the mass fraction of DMF in mixed solvent was 0.9 . The results are shown in Table III. When the solvent-to feed ratio is $6: 1$, the maximum relative volatility is 1.162 . What's more, the trend that the gradient of variation of relative volatility is similar to the results the COSMO-RS model calculated.

TABLE III: EXPERIMENTAL DATA OF RELATIVE VOLATILITY OF CP AND NH AT DIFFERENT SOLVENT-TO-FEED RATIOS

\begin{tabular}{cc}
\multicolumn{2}{c}{ AT DIFFERENT SOLVENT-TO-FEED RATIOS } \\
\hline \hline Solvent-to-feed ratio & DMF : cyclohexanol = 9:1 \\
\hline $2: 1$ & 1.056 \\
$3: 1$ & 1.094 \\
$4: 1$ & 1.127 \\
$5: 1$ & 1.150 \\
$6: 1$ & 1.162 \\
\hline \hline
\end{tabular}

\section{CONCLUSION}

The objective was to develop a rapid way to screen effective solvents for separation of cyclopentane and neohexane system by extractive distillation. COSMO-RS can predict the infinite dilution activity coefficients of alkanes and cycloalkanes in different solvents, but not always give reliable results. Experimental data are the most reliable and best source of thermodynamic properties. Comparing the available experimental data of IDAC with the predicted results of COSMO-RS, different empirical relationships for the alkane-solvent and cycloalkane-solvent IDACs in terms of COSMO-RS predictions were developed to obtain much more accurate IDACs, which provide higher level of accuracy for solvent screening. Among three preselected solvents, DMF was the best solvent candidate. As the solvent-to-feed ratio and the basic solvent and cosolvent ratio changed, the performances of mixed solvent (DMF + cyclohexanol) were also studied by the COSMO-RS model. Experimentally, when the solvent-to-feed ratio was 6:1, with $\mathrm{DMF}+$ cyclohexanol mixed solvent, the relative volatility changed to 1.162 , meanwhile, the variation trend of relative volatility with solvent-to-feed ratio is similar to the COSMO-RS predictions. In this sense, the COSMO-RS model can be used for solvent screening in extractive distillation.

\section{REFERENCES}

[1] X. Xu, "Development and production of cyclopentane foaming agent," Guangzhou Chemical Industry, vol. 40, pp. 51-60, 2013.

[2] X. Li, "Application of cyclopentane combined polyether in solar water heater," Chemical Propellants \& Polymeric Materials, vol. 13, pp. 75-78, 2015.

[3] M. M. Hicks-Bruun, J. H. Bruun, and W. B. Mason Faulconer, "Isolation of 2, 2-dimethylbutane from natural gas and determination of its physical properties," J. Am. Chem. Soc., vol. 61, pp. 3099-3101, 1939.

[4] K. T. Serijan, R. A. Spurr, and L. C. Gibbons, "The system cyclopentane-neohexane-aniline," J. Am. Chem. Soc., vol. 68 pp. 1763-1764, 1946

[5] C. R. Gardner, "Separation of cyclopentane-neohexane mixtures by azeotropic distillation with methyl formate" U.S. Patent 2498 928, Feb. $28,1950$.

[6] R. E. Brown and F. M. Lee, "Extractive distillation of hydrocarbon feeds employing mixed solvent" U.S. Patent 4954 224, Sept. 4, 1990.

[7] M. Lavanya, M. N. Suresh, B. Sairam. "Effect of solvents for the production of cyclopentane by extractive distillation," Petrol. Sci. Technol., vol. 25, pp. 1593-1603, 2007.

[8] A. Klamt, F. Eckert and W. Arlt, "COSMO-RS: An alternative to simulation for calculating thermodynamic properties of liquid mixtures," Annual Review of Chemical and Biomolecular Engineering, vol. 1, pp. 101-122, 2010.

[9] A. Klamt, "The COSMO and COSMO-RS solvation models," Wiley Interdisciplinary Reviews: Computational Molecular Science, vol. 1, pp. 699-709, 2011.

[10] A. Klamt, "Conductor-like screening model for real solvents: A new approach to the quantitative calculation of salvation phenomena," $J$. Phys. Chem., vol. 99, pp. 2224-2235, 1995.

[11] R. Putnam, R. Taylor, A. Klamt, F. Eckert, M. Schiller, "Prediction of infinite dilution activity coefficients using COSMO-RS," Ind. Eng. Chem. Res., vol. 42, pp. 3635-3641, 2003.

[12] P. M. Mathias, J.R. Elliott Jr, A. Klamt, "Butadiene purification using polar solvents. Analysis of solution nonideality using data and estimation methods," Ind. Eng. Chem. Res., vol. 47, pp. 4996-5004, 2008.

[13] D. Tiegs et al., Activity coefficients at infinite dilution. Part 1, C(1)-C(9). Frankfurt: DECHEMA, 1986.

[14] R.L. David, CRC Handbook of Chemistry and Physics CD-ROM, Version 2010. Boca Raton: CRC Press, 2009. 


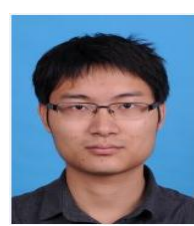

Lehuan Wu was born in Zhejiang province, China in 1987. He received his bachelor degree in 2009 from China University of Petroleum (Beijing). He is now a phD student in China University of Petroleum (Beijing) under the supervision of Professor Yansheng Liu. His current research interests include extractive distillation design, the thermodynamics property of ionic liquids and molecular dynamic simulation for clathrate hydrates.

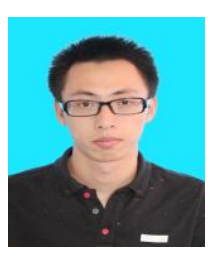

Xinyun Pu was born in Jiangsu province, China in 1990. He received his bachelor degree in 2013 from China University of Petroleum (Beijing). He is now a PhD student in China University of Petroleum (Beijing) under the supervision of Professor Yansheng Liu. His current research interests include separation process in chemical engineering and predictive molecular thermodynamics.

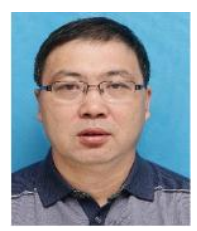

Yansheng Liu was born in Hebei province, China in 1963. He received his Ph.D degree in 2012 from China University of Petroleum (Beijing). He is now a professor in China University of Petroleum (Beijing).He has received two China's Petroleum Industry Associationa Technology Progress Prizes (first class), and one Minstry of Educational Science and Technolofy Progress Prize (first class). His main research interests are transfer and separation process in chemical engineering and equipment development and trouleshooting. He has contributed to more than 20 papers in international journals. 